\title{
Hemólisis severa postcierre de leak perivalvular mitral por vía transapical que requirió reintervención por vía transeptal
}

\author{
Severe hemolysis after transapical closure of mitral perivalvular leak \\ requiring transeptal reintervention
}

Marcelo A. Agüero', Jorge A. Baccaro', Pablo D. Liva', Gastón Pozzi', Walter J. García²

\section{RESUMEN}

Las manifestaciones más frecuentes de las fugas perivalvulares son la insuficiencia cardíaca (IC) y la hemólisis. Reportamos un caso de cierre de leak perivalvular mitral por vía transapical, en paciente con síntomas de insuficiencia cardíaca que posteriormente desarrolló severa hemólisis secundaria a fuga residual pequeña con jet de alta velocidad y su reintervención posterior por vía transeptal.

Palabras claves: válvula mitral, hemólisis, leak mitral perivalvular.

\section{ABSTRACT}

Congestive Heart Failure and hemolysis are the most common presentation of perivalvular leaks. We report a case of transapical closure of a Mitral perivalvular leak in a patient with Heart Failure who lately developed severe hemolysis due to a small residual defect and high-velocity jet, and its reintervention using transeptal approach.

Keywords: mitral valve, hemolysis, mitral perivalve leak.

Revista Argentina de Cardioangiología Intervencionista 2020;11(2):73-76. https://doi.org/10.30567/RACI/202002/0073-0076

\section{INTRODUCCION}

Los leaks o fugas perivalvulares (LPV) se presentan en un 6 a $15 \%$ de las cirugías de reemplazo valvular. Son más frecuentes en posición mitral que en aórtica y clásicamente su aparición se halla relacionada a condiciones tales como friabilidad de tejidos, calcificación o infecciones ${ }^{1,2}$. La presencia de LPV moderados o severos luego del reemplazo valvular quirúrgico o percutáneo se asocia a aumento de la mortalidad ${ }^{3}$. En casos de regurgitaciones moderadas o severas la manifestación habitual es la insuficiencia cardíaca. LPV pequeños que producen jets de alta velocidad pueden producir anemia hemolítica como manifestación principal ${ }^{4}$. La endocarditis infecciosa ha sido también descripta como una manifestación infrecuente. Las condiciones anatómicas predisponentes al desarrollo de LPV dificultan la resolución quirúrgica ulterior. Por otra parte, estos pacientes presentan un perfil de riesgo elevado para una recirugía ya que la presencia de IC o hemólisis severa compromete su estado general. En este contexto, la terapia transcatéter se ha convertido en una opción de creciente interés para el cierre de LPV.

Reportamos un caso de cierre de LPV mitral paraseptal por vía transapical en un paciente sintomático por IC que en el seguimiento desarrolla severa hemólisis secundaria a leak residual pequeño, requiriendo reintervención por vía transeptal.

1. Servicio de Cardiología Intervencionista.

2. Servicio de Ecocardiografía. Instituto de Cardiología de Corrientes "Juana F. Cabral". Corrientes. Argentina.

$\triangle$ Correspondencia: Marcelo A. Agüero. Bolívar 1334. W3400AMZ Corrientes. Rep.Argentina.Tel/Fax:03794410000.marceloaguer@gmail.com

Los autores declaran no tener conflictos de intereses.

Recibido:08/04/2020|Aceptado: 28/04/2020

\section{CASO CLÍNICO}

Paciente de 72 años, hipertensión arterial (HTA), diabetes mellitus (DBT) 2, infarto de miocardio previo, portador de enfermedad coronaria de tronco y 3 vasos con insuficiencia mitral moderada a severa de etiología mixta. Es sometido a cirugía de bypass coronario y de reemplazo valvular mitral con prótesis biológica nro. 29 , sin complicaciones. A los 3 meses consulta por disnea progresiva y signos de IC congestiva. Se realiza eco-Doppler cardíaco constatándose insuficiencia mitral 3 secundaria a leak perivalvular.

Evoluciona con IC de difícil manejo farmacológico y es siendo internado por descompensación hemodinámica. Se planifica el cierre percutáneo del defecto para lo cual se realiza eco-transesofágico (ETE) y tomografía computarizada (TAC) volumétrica (Figura 1).

Dada la localización paraseptal se decide el abordaje transapical, para lo cual se procede a realizar anestesia general, intubación orotraqueal y colocación de sonda transesofágica. Se accede al ápex del VI a través de incisión en el 5 to espacio intercostal izquierdo, línea axilar anterior, según parámetros derivados de la evaluación con TAC. Se punza el ápex con aguja $18 \mathrm{G}$ y se coloca introductor valvulado 6 Fr (Cordis). Con guía radioscópica y ultrasonográfica se lleva catéter JR 5 Fr (Cordis) hasta la zona del defecto, y se progresa a través del mismo hasta aurícula izquierda con guía hidrofílica 0,032" (Terumo). Se avanza vaina Occlutech 9 Fr, dejando a través del defecto guía coronaria HTFII (Abbott) como protección de eventual pérdida de la posición alcanzada. Por dentro de la vaina se lleva dispositivo oclusor OCCLUTECH PLD 14W. Se avanza el primer disco a AI, se retira la vaina a VI y se conforma el segundo disco en VI. Se constata resultado adecuado con disminución de la regurgitación a trazas por ETE, por lo que se decide liberar el dispositivo (Figura 2). Finaliza el procedimiento con hemosta- 

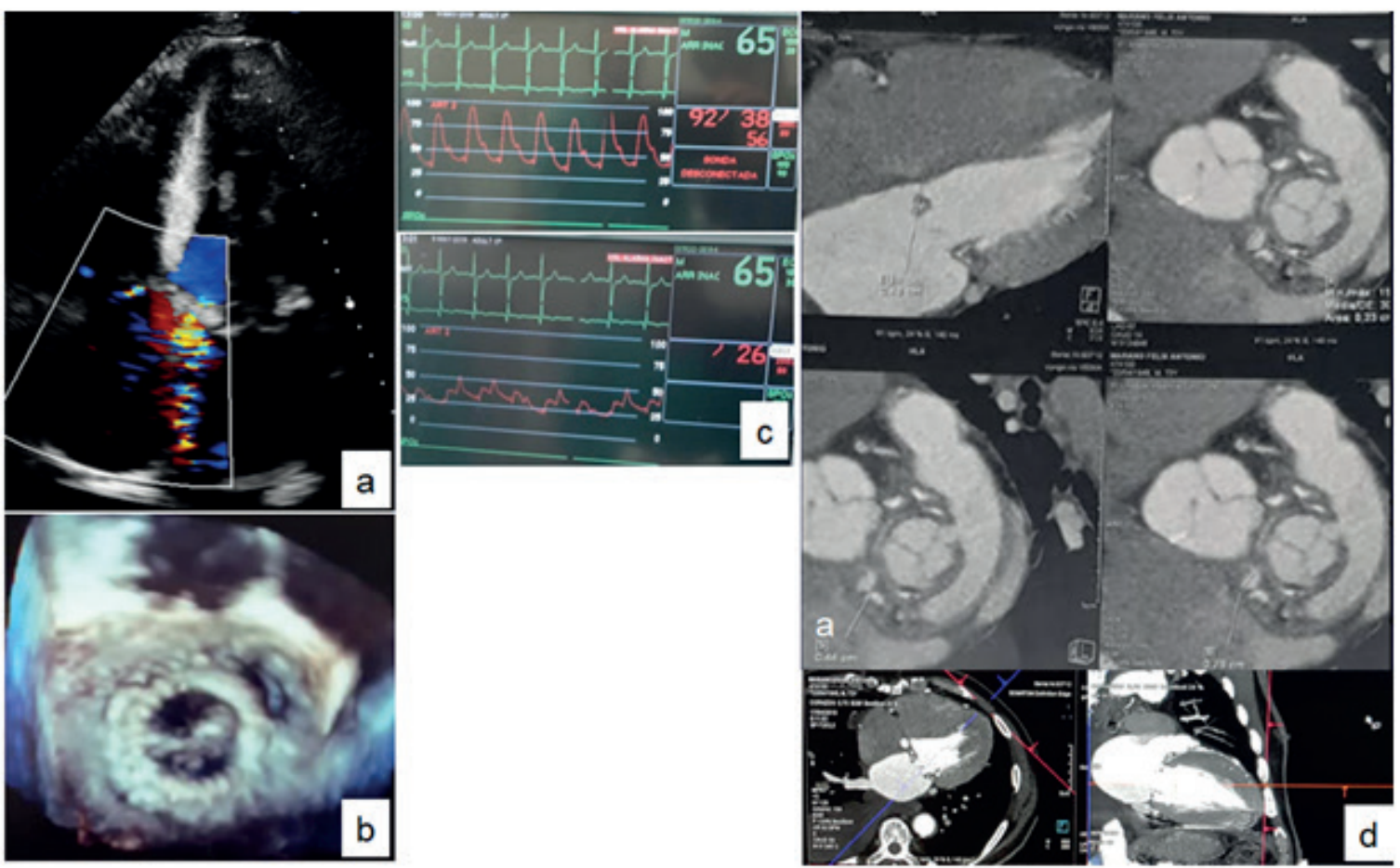

Figura 1. Evaluación previa al cierre de la fuga por vía transapical. a) Eco-Doppler: jet de IM paraseptal. b) ETE 3D "en face": leak de 12 mm de diámetro mayor en hora 2. c) Repercusión hemodinámica: PAP 92/38 (56) $\mathrm{mmHg}$, onda v $53 \mathrm{mmHg}$. d) VCT: evaluación del leaky del acceso transapical.

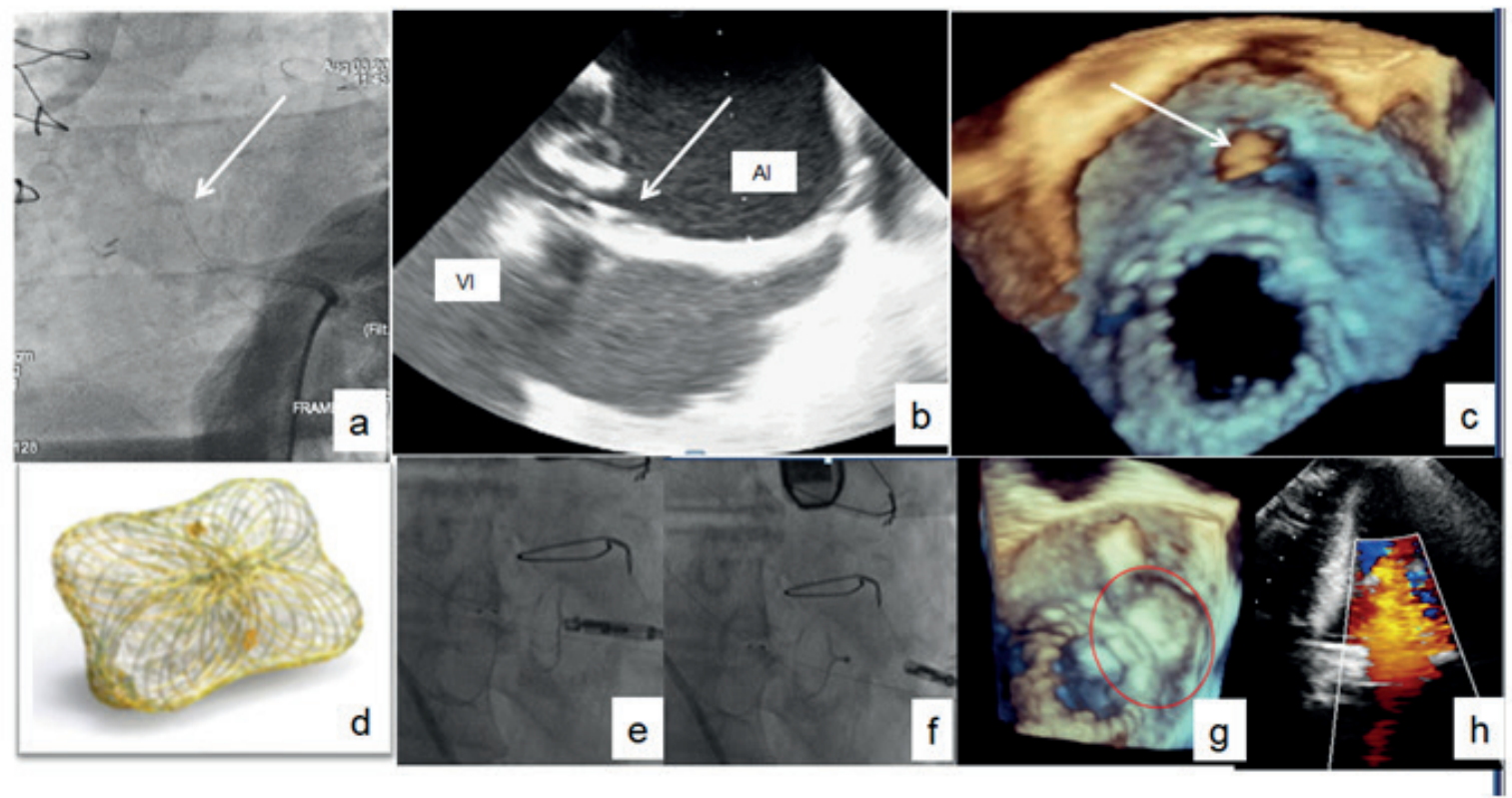

Figura 2. (ierre del leak por vía transapical. a) y b) Radioscopia y ETE: quía coronaria HTF2 a través del defecto (flecha) desde VI hacia Al. c) ETE 3D "en face" confirmando el pasaje del alambre guía (flecha) a través del leak. d) Dispositivo oclusor Occlutech PLD 14W. e) Dispositivo conectado al sistema de liberación.f) Dispositivo liberado. g) ETE 3D "en face": dispositivo normoposicionado (círculo). h) Eco-Doppler: reducción a trazas de la fuga.

sia quirúrgica, sin complicaciones. El paciente es externado al 3er día postintervención.

Evoluciona favorablemente al alta, reduciéndose progresivamente la dosis de diuréticos. A los 45 días posprocedimiento consulta nuevamente por disnea, astenia y pérdida de peso. Se constata en el laboratorio: hematocrito (Hto) 18\%, hemoglobina $6,4 \mathrm{~g} / \mathrm{dl}$, bilirrubina total (BT) 4,27 mg/dl, directa (BD) $2,46 \mathrm{mg} / \mathrm{dl}$, indirecta (BI) $1,82 \mathrm{mg} / \mathrm{dl}$, haptoglobina $4 \mathrm{mg} / \mathrm{dl}$. Se interpreta el cuadro como anemia hemolítica intravascular.
Se realiza eco-Doppler constatándose fuga residual pequeña con jet regurgitante de alta velocidad. Durante las semanas siguientes es politransfundido y evoluciona con deterioro progresivo del estado general. Se completa evaluación con ETE y TAC volumétrica para planear la reintervención. Se identifica la localización precisa del defecto, entre el disco ventricular del dispositivo previamente implantado y una porción calcificada del anillo mitral (Figura 3).

Se decide, debido al deterioro del estado general del paciente 

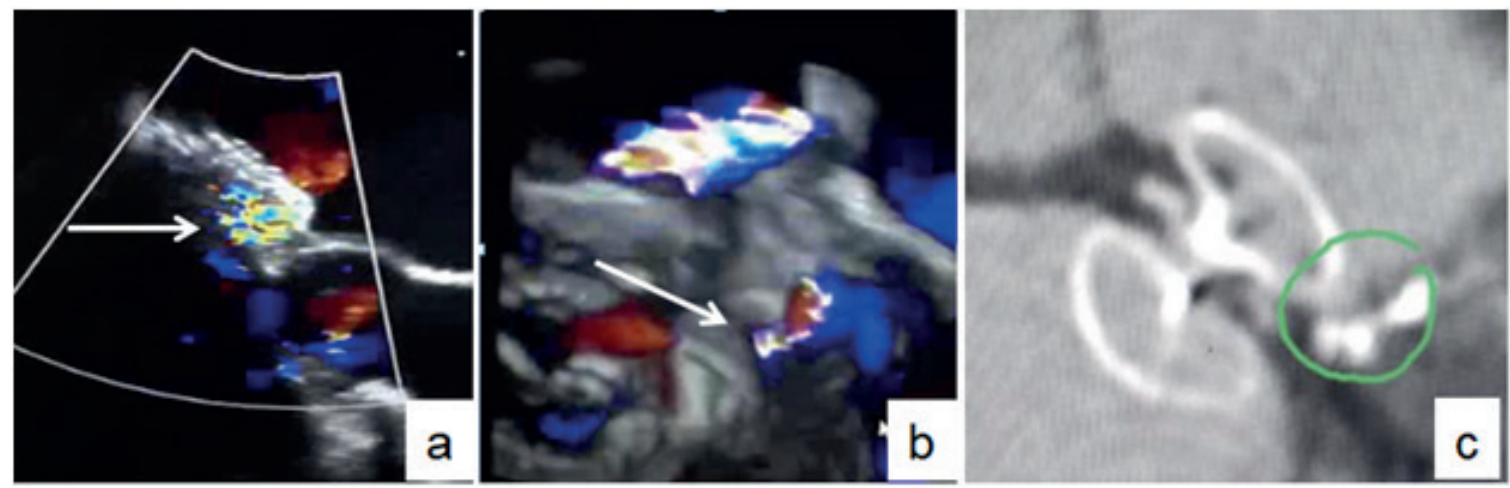

Figura 3. Identificación del jet residual de alta velocidad en ETEy VCT. a) ETE: jet de alta velocidad produciendo "aliasing" (flecha). c) VCT: identificación precisa del sitio de fuga, en relación con porción calcificada del anillo mitral
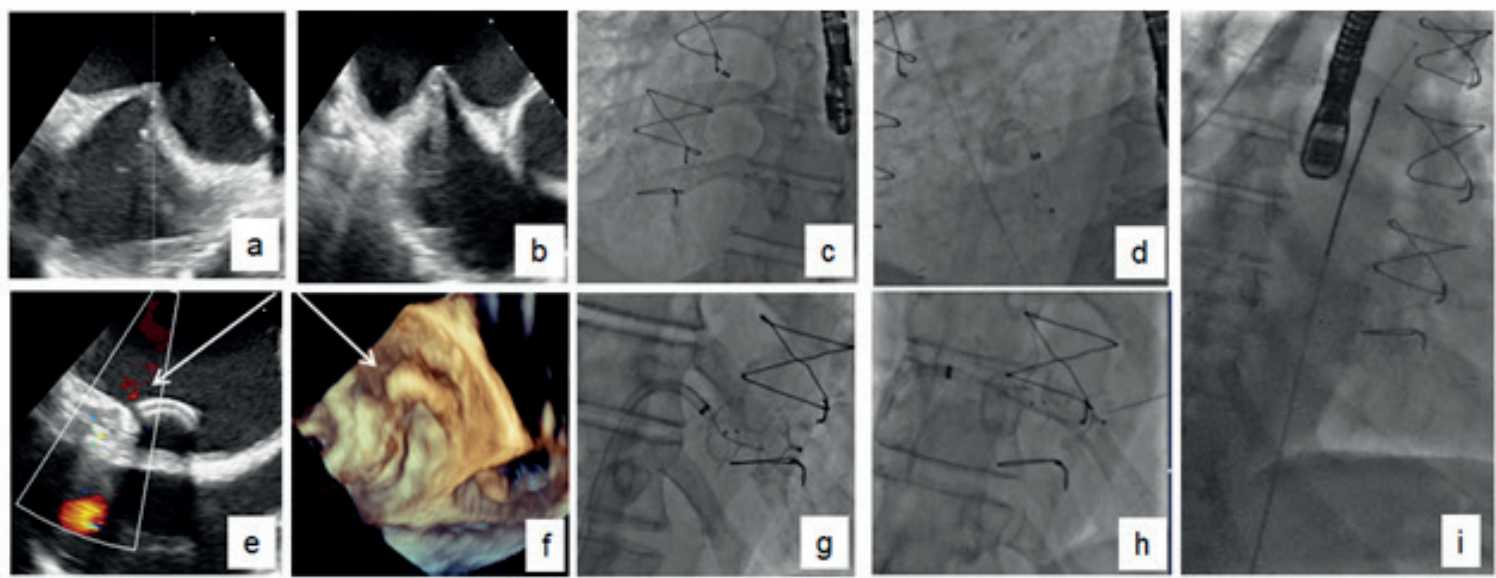

Figura 4. Reintervención por vía transeptal. a) y b) ETE: ejes bicavo y corto, respectivamente. "Tenting" sobre el septum interauricular. c) y d) Vaina deflectable Fustar en posición neutra y en máxima deflexión, repectivamente. e) y f) Posición de la vaina Fustar próxima al defecto en ETE 2D y 3D, respectivamente (flechas). g) y h) Pasaje de guía Command 18 a través del defecto. i) Luego de haber capturado con lazo la guía Command en VI, la misma es llevada a aorta y luego exteriorizada por introductor arteria femoral.
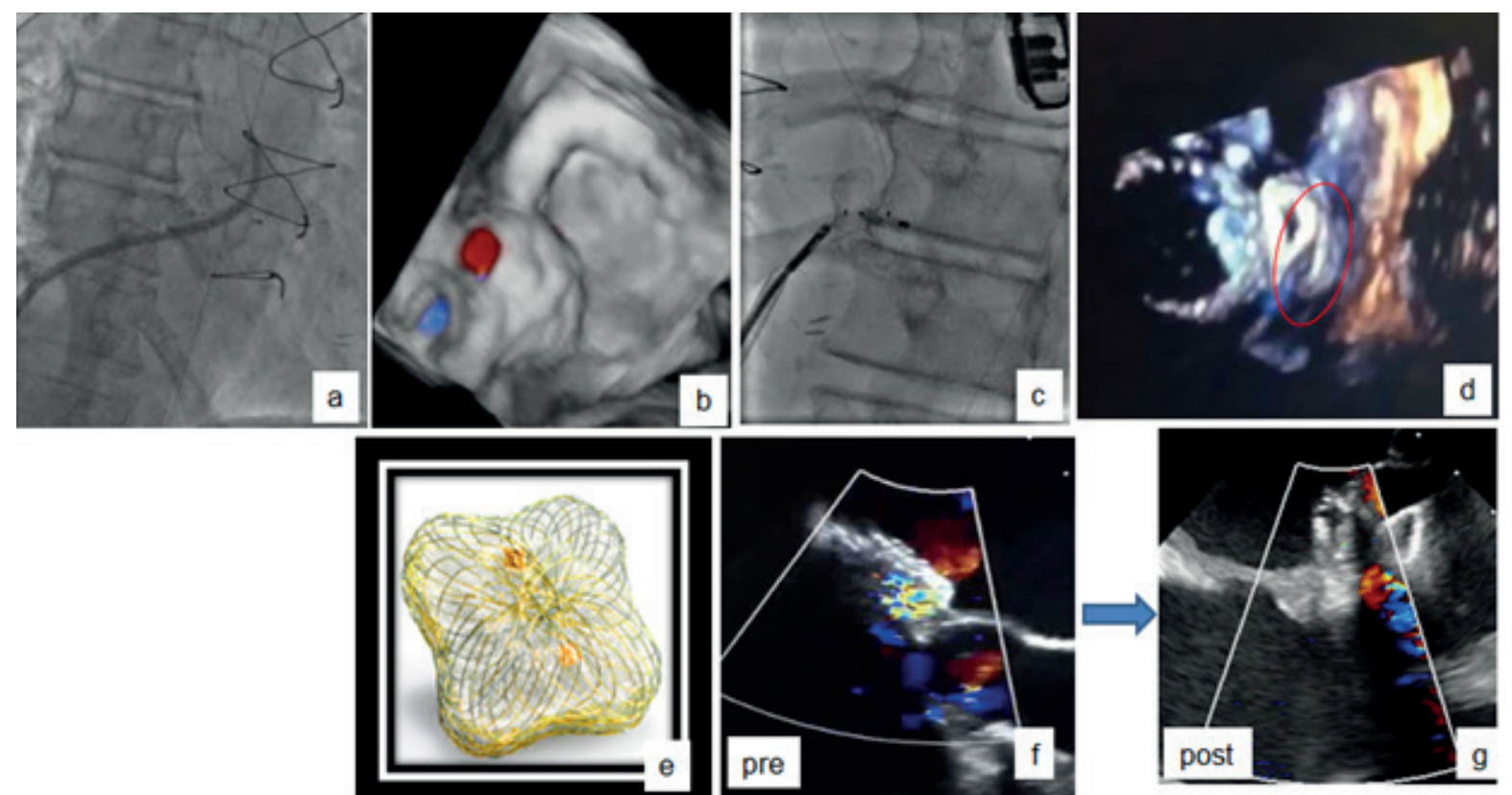

Figura 5. Reintervención por vía transeptal (cont.). a) Vaina Occlutech $9 F$ a través del defecto. b) ETE 3D color confirmando el pasaje de la vainapor fuera del dispositivo previamente implantado. c) Dispositivo oclusor Occlutech PLD 5T en posición, previo a la liberación. d) Eco 3D confirmanfo la posición adecuada del dispositivo oclusor. e) Dispositivo oclusor OcclutechPLD 5T.f) y g)Comparación de flujos a través del defecto antes y al finalizar el procedimiento, respectivamente. 
y a pesar de la localización desfavorable del defecto, evitar repetir el acceso transapical e intentar primariamente el abordaje transeptal.

Bajo anestesia general se procede a punzar vena femoral derecha. Se realiza punción transeptal guiada por ETE, con aguja de Brockenbrough, seleccionando porción posterosuperior del septum interauricular. Se avanza a AI vaina deflectable Fustar 8 Fr (Lifetech). Con rotación antihoraria y deflexión máxima de la misma se consigue posicionar su extremo distal próxima al defecto, según imágenes obtenidas en ETE 3D. Luego de múltiples intentos se consigue avanzar al VI una guía Command-18 de $300 \mathrm{~cm}$ (Abbott). Se intenta avanzar sobre la misma distintos dispositivos (catéteres JR y MP 4 F y balones OTW), sin éxito. Se decide punzar arteria femoral y colocar introductor valvulado $6 \mathrm{Fr}$ (Cordis) y llevar lazo hasta el VI donde se captura la guía Command-18 y se la exterioriza a través del introductor arterial (Figura 4). Con el soporte así obtenido se consigue avanzar vaina liberadora $9 \mathrm{Fr}$ al VI. Por dentro de la misma, y dejando la guía en paralelo, se avanza dispositivo OCCLUTECHPLD 5T, se retrae la vaina con lo que se despliega el disco ventricular y luego el auricular. Se comprueba por ETE cese total del flujo a través del leak por lo que se libera el dispositivo (Figura 5). Finaliza el procedimiento sin complicaciones. Con evolución intrahospitalaria sin eventos, es dado de alta a las $48 \mathrm{hs}$. En el seguimiento luego del alta se constata rápida mejoría clínica y, en el laboratorio a los 30 días, mejoría de la anemia y de los parámetros de hemólisis: Hto 29\%, BT 0,55 $\mathrm{mg} / \mathrm{dl}, \mathrm{BD} 0,23 \mathrm{mg} / \mathrm{dl}, \mathrm{BI} 0,32 \mathrm{mg} / \mathrm{dl}$.

\section{DISCUSIÓN}

El análisis del caso descipto permite algunas reflexiones: La evaluación con imágenes multimodales (eco-Doppler, ETE 3D, VCT y radioscopia) es imprescindible en el diagnóstico y planificación del tratamiento de LPV. Por lo tanto, y como sucede con el tratamiento de otras cardiopatías estructurales, la formación del cardiólogo intervencionista en las mencionadas técnicas de imágenes es necesaria para el abordaje de esta patología 5 . Los LPV mitrales paraseptales representan un desafío técnico significativo por la dificultad de acceso al mismo por vía transeptal. Este hecho motivó en nuestro paciente la intervención inicial por vía transapical. Sin embargo, en la reintervención, y motivados por el mayor deterioro clínico expe-

\section{REFERENCES}

1. Ionescu A, Fraser AG, Butchart EG. Prevalence and clinical significance of incidental paraprostheticvalvar regurgitation: a prospective study using transoesophageal echocardiography. Heart 2003;89:1316-21.

2. O'Rourke DJ, Palac RT, Malenka DJ, Marrin CA, Arbuckle BE, Plehn JF. Outcome of mild periprosthetic regurgitation detected by intraoperative transesophageal echocardiography. J Am CollCardiol 2001;38:163-6.

3. Sponga S, Perron J, Dagenais F, et al. Impact of residual regurgitation after aortic valve replacement. Eur I CardiothoracSurg 2012;42:486-92.

4. Eleid MF, Cabalka AK, MaloufJF, Sanon S, Hagler DJ, Rihal CS. Techniques and Outcomes for the Treatment of Paravalvular Leak. CircCardiovasc Interv2015;8:e001945. rimentado por el paciente, decidimos intentar primariamente la vía transeptal. Luego del resultado satisfactorio obtenido por esta vía podemos concluir que las localizaciones más desafiantes (como es el caso de fugas paraseptales) pueden ser abordadas por vía transeptal. La obtención de imágenes adecuadas en la sala (ETE 3D), la disponibilidad de materiales (vainas deflectables, guías, lazos, etc.) y el manejo técnico de dichos materiales permiten intentar primariamente la vía transeptal en virtualmente todas las localizaciones.

Habitualmente se define como éxito del procedimiento de cierre percutáneo de LPV a la reducción de la regurgitación a grado $\leq 1$ en ausencia de complicaciones. Este resultado se obtiene en el 70-90\% de los casos y aumenta con el incremento en la experiencia de los operadores. Actualmente existen reportes de éxito técnico del $90 \%$ incluso en los casos mas complejos $^{6,7}$. El caso aquí reportado, a pesar de la reducción significativa de la regurgitación en la primera intervención a $\leq 1$, condicionó el desarrollo de anemia hemolítica severa que deterioró rápidamente al paciente y obligó a la reintervención al poco tiempo. Por lo tanto, si bien en pacientes con LPV que desarrollan insuficiencia cardíaca el objetivo de reducir la regurgitación a $\leq 1$ es lógico, no se debe subestimar la importancia clínica que pueden producir jets residuales de alta velocidad.

\section{CONCLUSIONES}

El diagnóstico y la planificación del tratamiento percutáneo de LPV, como en el tratamiento de otras cardiopatías estructurales, requiere entrenamiento en imágenes multimodales por parte del cardiólogo intervencionista.

El cierre de LPV mitrales paraseptales es técnicamente demandante. Defectos en esta localización pueden abordarse por vía transapical o transeptal. La elección de esta última es atractiva para reducir morbilidad en pacientes críticos pero conlleva la necesidad de gran diversidad de material específico y gestos técnicos avanzados.

Finalmente, se debe procurar alcanzar el menor grado de regurgitación posible para asegurar una evolución clínica favorable.

\section{AGRADECIMIENTOS}

Dr. León Valdivieso. Fundación Favaloro. Dr. Walter Bonfanti.
5. Sorajja P, Cabalka AK, Hagler DJ, Rihal CS. The learning curve in percutaneous repair of paravalvular prosthetic regurgitation: an analysis of 200 cases. JACC Cardiovasc Interv2014;7:521-9.

6. Ruiz CE, Jelnin V, Kronzon I, et al. Clinical outcomes in patients undergoing percutaneous closure of periprostheticparavalvular leaks. J Am Coll Cardiol2011;58:2210-7. 